\title{
Symbol Timing Recovery for GMSK Modulation Based on Squaring Algorithm
}

\author{
Yik-Chung Wu, Student Member, IEEE, and Tung-Sang Ng, Senior Member, IEEE
}

\begin{abstract}
A new all-digital symbol timing estimator for GMSK signals is proposed. It is based on the squaring algorithm and has a feedforward structure. Performance in AWGN channel is assessed by computer simulation, and is compared with the modified Cramer-Rao bound and other existing algorithms. The performance of the proposed estimator is shown to be close to that of ML algorithm.
\end{abstract}

Index Terms-GMSK, squaring algorithm, symbol timing recovery.

\section{INTRODUCTION}

YMBOL timing recovery is a crucial part in the detection of Gaussian minimum shift keying (GMSK) signals. Several all-digital algorithms have been proposed [1]-[3]. In [1], the symbol timing recovery algorithm requires the transmission of a preamble, which reduces the transmission efficiency. The algorithm introduced in [2] is based on the maximum likelihood method. Although it provides good performance, the implementation complexity is large. An ad hoc approach is proposed in [3], but the performance is rather poor.

In this letter, we propose a new all-digital symbol timing recovery algorithm for GMSK signals based on the squaring algorithm. The squaring algorithm is originally proposed for linear modulations [4] and has not been used for GMSK signals. The resultant estimator has a feedforward structure so that it is hang-up free and is suitable for implementation in software radio.

\section{Direct ApPlication of SQUaring Algorithm TO GMSK SIGNAL}

Let the sequence $\left\{r_{k}\right\}$ represents the signal obtained by sampling the complex envelope of a linear modulation at rate $1 / T_{s}=N / T$ where $T$ is the symbol transmission duration and $N$ is the oversampling ratio. The symbol timing delay $\varepsilon$ can be estimated by computing the complex Fourier coefficients at the symbol rate for every segment of $L_{0} N$ samples ( $L_{0}$ symbols) of $\left|r_{k}\right|^{2}$. That is, the estimate $\hat{\varepsilon}_{m}$ is given by [4]

$$
\hat{\varepsilon}_{m}=-\frac{1}{2 \pi} \times \arg \left(\sum_{k=m L_{o} N}^{(m+1) L_{o} N-1}\left|r_{k}\right|^{2} e^{-j 2 \pi k / N}\right)
$$

Manuscript received December 4, 2000. The associate editor coordinating the review of this letter and approving it for publication was Dr. N. Van Stralen. This work was supported by the Hong Kong Research Grants Council and by the University Research Committee of The University of Hong Kong, Hong Kong.

The authors are with the Department of Electrical and Electronic Engineering, University of Hong Kong, Pokfulam Road, Hong Kong (e-mail: ycwu@eee.hku.hk; tsng@eee.hku.hk).

Publisher Item Identifier S 1089-7798(01)04508-2. where $\arg (x) \in\{-\pi, \pi\}$ denotes the phase of $x$ and $\hat{\varepsilon}_{m} \in$ $\{-0.5,0.5\}$.

Notice that if the original symbol timing delay $\varepsilon$ is not within the range $\{-0.5,0.5\}$, it actually corresponds to the case when the signal is shifted more than one symbol time. The estimate $\hat{\varepsilon}_{m}$ will then be given by the original timing delay $\varepsilon$ add or subtract by an integer such that the result fall into the range $\{-0.5,0.5\}$.

The squaring operation is equivalent to self-convolution of signal spectrum in the frequency domain. The spectrum at the output of the squaring operation contains spectral lines at $f=$ $\pm 1 / T$, which give the timing information. The strength of these spectral lines depends on the degree of spectral overlap when the signal spectrums are $1 / T$ apart.

For GMSK signals, this symbol timing estimation algorithm is not necessarily effective since GMSK is a nonlinear modulation. However, it can be shown [5] that the complex envelope of the GMSK signal can be approximated by a linear modulation with a pulse shape $C_{o}(t)$. That is, the GMSK signal $s(t)$ can be approximated by

$$
\begin{aligned}
s(t) \approx & \sum_{N=0}^{\infty} \exp \left[j \pi h \sum_{n=0}^{N} a_{n}\right] C_{0}(t-N T-\varepsilon T) \\
= & \sum_{k=0}^{\infty} b_{2 k+1} C_{0}\left(t-2 k T-T-\varepsilon^{\prime}(2 T)\right) \\
& +j \sum_{k=0}^{\infty} b_{2 k} C_{0}\left(t-2 k T-\varepsilon^{\prime}(2 T)\right)
\end{aligned}
$$

where

$$
C_{o}(t)=\prod_{n=0}^{3} \frac{\sin [\Psi(t+n T)]}{\sin (h \pi)}
$$

with

$$
\Psi(t)= \begin{cases}\pi \int_{-\infty}^{t} g(\tau) d \tau, & t<L T \\ h \pi-\pi \int_{-\infty}^{t-L T} g(\tau) d \tau, & t \geq L T\end{cases}
$$

and $b_{2 k}=a_{2 k} b_{2 k-1}, b_{2 k+1}=-a_{2 k+1} b_{2 k}$ and $b_{-1}=1$. In these expressions, $a_{n}$ is the transmitted data of the GMSK signal, $g(t)$ is the convolution between a Gaussian pulse and a rectangular pulse, and $\varepsilon$ is the symbol timing delay of the GMSK signal with $\varepsilon=2 \varepsilon^{\prime}$. Despite the linear approximation indicates the possibility of symbol timing estimation based on the squaring method, simulation results indicate that the squaring method may not work for GMSK signals. This is due to the small 
spectral density of $C_{o}(f)$ for $|f|>0.5 / T$. When the signal spectrums are $1 / T$ apart, the spectral overlapping is not large enough to generate a stable estimate.

As the squaring method is commonly used in linear modulation with raised cosine pulse, one method to enhance the frequency components of $C_{o}(f)$ for $|f|>0.5 / T$ is to design a matched filter such that

$$
H_{M F}(f)=R C_{2 T}(f) / C_{o}(f)
$$

where $H_{M F}(f)$ is the frequency response of the matched filter and $R C_{2 T}(f)$ is the spectrum of the raised cosine pulse with $3-\mathrm{dB}$ cutoff frequency $f_{c}=1 / 2 T$. For the case of 0.3 GMSK, the maximum value of the excess bandwidth $\alpha$ of the raised cosine pulse in (3) is 0.2 due to the fact that if $\alpha$ is greater than 0.2 , there are some frequencies in which $R C_{2 T}(f)$ is finite but $C_{o}(f)$ is zero, making $H_{M F}(f)$ infinite at these frequencies. With this matched filter, direct application of the squaring method is possible, but the performance is not satisfactory. This motivates the proposed timing estimator.

\section{The Proposed TIMING Estimator}

Timing information can also be obtained by viewing a GMSK signal as a combination of two orthogonal linear modulations each with symbol rate $1 / 2 T$ and staggered with a time $T$ as given in (2). Timing delays can then be estimated separately in inphase and quadrature channels and the two estimates can be subsequently combined to give the timing delay estimate for the GMSK signal, $\hat{\varepsilon}$.

Ignore the estimation variance at this moment (in the case of high SNR or large $L_{\circ}$ ), the timing estimates from the inphase and quadrature branch in (2), denoted by $\hat{\varepsilon}_{I}$ and $\hat{\varepsilon}_{Q}$ respectively, are

$$
\hat{\varepsilon}_{I}=0.5+\varepsilon^{\prime} \quad \text { and } \quad \hat{\varepsilon}_{Q}=\varepsilon^{\prime} .
$$

Without loss of generality, $\varepsilon^{\prime} \in\{-0.5,0.5\}$ is assumed. If $0.5 \geq \varepsilon^{\prime}>0, \hat{\varepsilon}_{I}$ will be greater than 0.5 , the estimate will be given by $0.5+\varepsilon^{\prime}$ subtracted by 1 such that the result is in the range $\{-0.5,0.5\}$, i.e.,

$$
\begin{aligned}
\hat{\varepsilon}_{I} & =0.5+\varepsilon^{\prime}-1=\varepsilon^{\prime}-0.5 \\
\hat{\varepsilon}_{Q} & =\varepsilon^{\prime}
\end{aligned}
$$

and it follows that

$$
\left|\hat{\varepsilon}_{I}\right|+\left|\hat{\varepsilon}_{Q}\right|=\left|\varepsilon^{\prime}-0.5\right|+\left|\varepsilon^{\prime}\right|=0.5-\varepsilon^{\prime}+\varepsilon^{\prime}=0.5 .
$$

If $-0.5 \leq \varepsilon^{\prime} \leq 0$, both $0.5+\varepsilon^{\prime}$ and $\varepsilon^{\prime}$ will be in the range of $\{-0.5,0.5\}$, and

$$
\left|\hat{\varepsilon}_{I}\right|+\left|\hat{\varepsilon}_{Q}\right|=\left|\varepsilon^{\prime}+0.5\right|+\left|\varepsilon^{\prime}\right|=0.5+\varepsilon^{\prime}-\varepsilon^{\prime}=0.5 .
$$

It can be concluded that $\left|\hat{\varepsilon}_{I}\right|+\left|\hat{\varepsilon}_{Q}\right|=0.5$. This means that when the value of $\left|\hat{\varepsilon}_{I}\right|$ increases, $\left|\hat{\varepsilon}_{Q}\right|$ decreases and vice versa.

Based on the above relationship, the proposed timing recovery method can be stated as

$$
\hat{\varepsilon}= \begin{cases}2 \hat{\varepsilon}_{I}, & \text { if }\left|\hat{\varepsilon}_{I}\right| \leq 0.25 \\ 2 \hat{\varepsilon}_{Q}, & \text { if }\left|\hat{\varepsilon}_{Q}\right|<0.25\end{cases}
$$

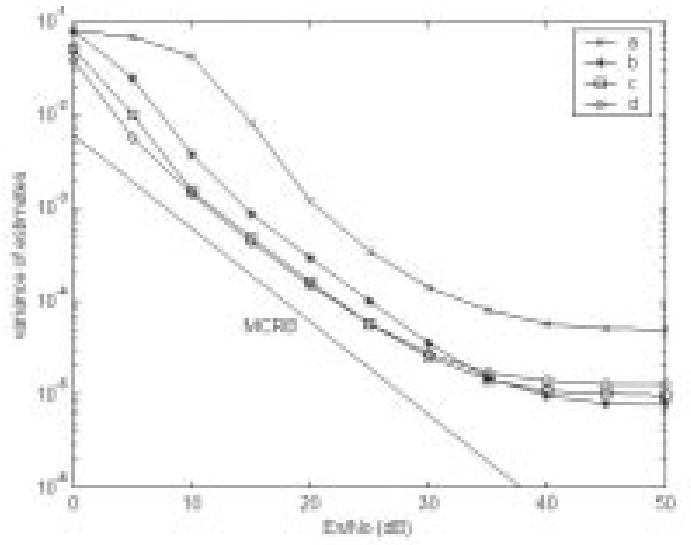

Fig. 1. Timing estimation variance comparison between (a) direct application of squaring method using matched filter $R C_{2 T}(f) / C_{o}(f)$ with $\alpha=0.2$ and $(\mathrm{b}, \mathrm{c}, \mathrm{d})$ the proposed timing estimator with different matched filters $[\mathrm{b}$ : $R C_{2 T}(f) / C_{o}(f)$ with $\alpha=0.2$, c: $R C_{4 T}(f) / C_{o}(f)$ with $\left.\alpha=1, \mathrm{~d}: C_{o}(f)\right]$.

There may be cases when both $\left|\hat{\varepsilon}_{I}\right|$ and $\left|\hat{\varepsilon}_{Q}\right|$ are very close to 0.25 , say $\left|\hat{\varepsilon}_{I}\right|=0.24$ and $\left|\hat{\varepsilon}_{Q}\right|=0.26$, and due to the variance of estimation, none of the case in (5) occur. In this situation, either $\hat{\varepsilon}_{I}$ or $\hat{\varepsilon}_{Q}$ can be chosen to give the estimate of the GMSK signal as they both give a value close to 0.5 or -0.5 . Note that there is no need to enhance any frequency component of $C_{o}(f)$ when applying the squaring algorithm to GMSK signals in the proposed way as the timing estimates are taken from a signal of lower data rate $(1 / 2 T)$. The spectral overlapping is large enough when the signal spectrums are $1 / 4 T$ apart.

The approximated GMSK signal in (2) also leads to other choices of the matched filter for the proposed estimator, apart from (3). As the symbol rate of the approximated linear modulation in both $I$ and $Q$ channels is $1 / 2 T$, it is apparent that the matched filter can be chosen with $H_{M F}(f)=R C_{4 T}(f) / C_{o}(f)$, where $R C_{4 T}(f)$ is the Fourier transform of a raised cosine pulse with $3 \mathrm{~dB}$ cutoff frequency $f_{c}=1 / 4 T$. Another obvious choice is $H_{M F}(f)=C_{o}(f)$, which matched to the pulse shape of the approximated GMSK signal.

\section{Simulation Results}

The performance of the proposed squaring algorithm for GMSK is evaluated by simulation. The variance of the estimates against $E_{s} / N_{o}$ is calculated using Monte Carlo simulations in AWGN channels with each point obtained by averaging over $10^{4}$ estimates. In all simulations, the modulation is $0.3 \mathrm{GMSK}$, the observation length $L_{o}$ is 64 and the oversampling factor $N$ is 4 .

Fig. 1 compares the variances of the direct application of squaring method and the proposed estimation algorithm. The modified Cramer-Rao bound (MCRB) [7], [8] is also plotted for reference. It can be seen that by using the matched filter $R C_{2 T}(f) / C_{o}(f)$ with $\alpha=0.2$, the performance of direct application of the squaring algorithm is rather poor (curve a) as indicated by the distance from the MCRB. The performance of the proposed estimator (curve $b$ ) is better and is closer to the MCRB. Simulation results also indicate that the performance of the proposed estimator by using $C_{o}(f)$ and $R C_{4 T}(f) / C_{o}(f)$ as 


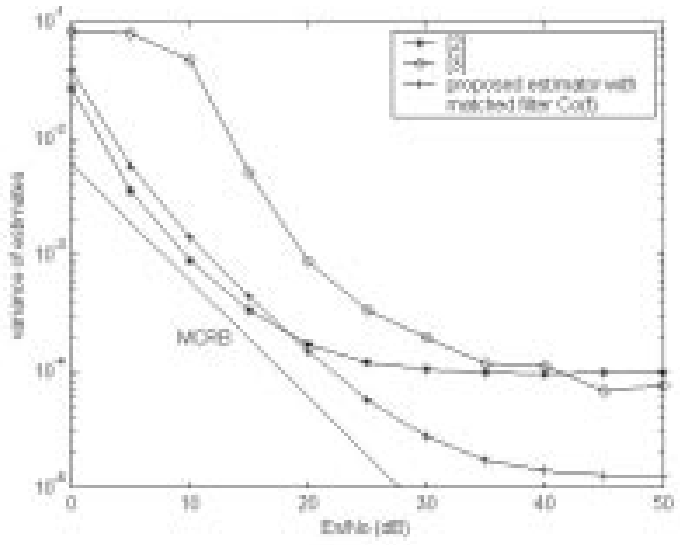

Fig. 2. Performance of the proposed estimator compared with other two existing algorithms.

matched filters are almost identical (curve $\mathrm{c}$ and $\mathrm{d}$ ). They offer better performances at low SNR compared with the case when $R C_{2 T}(f) / C_{o}(f)$ is used as the matched filter (curve b), while the later provides slightly lower variance of the estimate at high SNR.

The proposed estimator is also compared with two known algorithms: the algorithm of [2] and the estimator of [3] with the design parameter $M$ chosen to be 3. Fig. 2 illustrates the comparison results. It can be seen that the performance of the algorithm of [3] is relatively poor. When compared with the algorithm in [2], the proposed estimator exhibits only a limited degradation at low SNR but the variance of estimates from the proposed estimator is much smaller than that from [2] at high SNR. However, the proposed algorithm has a smaller implementation complexity. Since it is shown in [6] that, the computation of the complex Fourier coefficient at the symbol rate given by (1) can be realized by an IIR filter with a single coefficient of value -1 , the proposed algorithm requires only $2 L_{O} N$ real multiplications and $2 L_{o} N$ real additions per estimate. While the algorithm in [2] requires $3 L_{O} N+16$ real multiplications and $3 L_{o} N+12$ real additions per estimate, the reduction in complexity for the proposed algorithm is around $33 \%$. In the above calculation, multiplication with -1 or 2 does not count.

\section{CONCLUSIONS}

An all-digital timing estimator for GMSK modulation based on the squaring algorithm has been presented. The performance of the proposed estimator has been assessed in an AWGN environment by computer simulation. Simulation results have shown that direct application of the squaring algorithm to GMSK signals does not provide acceptable performance while the proposed estimator exhibits a performance closer to MCRB. The performance of the proposed estimator is close to that of ML algorithm. However, the proposed algorithm has a lower implementation complexity, implying lower power consumption, which is a critical requirement for mobile handset.

\section{REFERENCES}

[1] Y. L. Huang, K. D. Fan, and C. C. Huang, "A fully digital noncoherent and coherent GMSK receiver architecture with joint symbol timing error and frequency offset estimation," IEEE Trans. Veh. Technol., vol. 49, pp. 863-874, May 2000.

[2] M. Morelli and G. M. Vitetta, "Joint phase and timing synchronization algorithms for MSK-type signals," in Proc. Mini-Conf. Communication Theory 1999, June 1999, pp. 146-150.

[3] M. Morelli and U. Mengali, "Joint frequency and timing recovery for MSK-type modulation," IEEE Trans. Commun., vol. 47, pp. 938-946, June 1999.

[4] M. Oerder and H. Meyr, "Digital filter and square timing recovery," IEEE Trans. Commun., vol. 36, pp. 605-611, May 1988.

[5] Y.C. Wu and T. S. Ng, "New implementation of a GMSK demodulator in linear software radio receiver," in Proc. IEEE PIMRC2000, Sep. 2000, pp. 1049-1053.

[6] M. Rahnema, "Symbol timing recovery and tracking method for burst-mode digital communications," U.S. Patent 5870443.

[7] U. Mengali and A. N. D'Andrea, Synchronization Techniques for Digital Receivers. New York: Plenum, 1997.

[8] S. White and N. Beaulien, "On the application of Cramer-Rao and detection theory bounds to mean square error of symbol timing recovery," IEEE Trans. Commun., vol. 40, pp. 1635-1643, Oct. 1992. 